\title{
Quantitative sensing of microviscosity in protocells and amyloid materials using fluorescence lifetime imaging of molecular rotors
}

\author{
Alex J. Thompson ${ }^{1}$, T.-Y. Dora Tang' ${ }^{2}$ Therese W. Herling ${ }^{3}$, C. Rohaida Che Hak², Stephen Mann², \\ Tuomas P. J. Knowles ${ }^{3}$, Marina K. Kuimova ${ }^{1}$ \\ ${ }^{1}$ Department of Chemistry, Imperial College London, Exhibition Road, London, SW7 2AZ, UK \\ ${ }^{2}$ Centre for Organized Matter Chemistry, School of Chemistry, University of Bristol, Bristol, BS8 1TS, UK \\ ${ }^{3}$ Department of Chemistry, University of Cambridge, Lensfield Road, Cambridge, CB2 1EW, UK
}

*Corresponding author: alex.thompson08@imperial.ac.uk

\begin{abstract}
Molecular rotors are fluorophores that have a fluorescence quantum yield that depends upon intermolecular rotation. The fluorescence quantum yield, intensity and lifetime of molecular rotors all vary as functions of viscosity, as high viscosities inhibit intermolecular rotation and cause an increase in the non-radiative decay rate. As such, molecular rotors can be used to probe viscosity on microscopic scales. Here, we apply fluorescence lifetime imaging microscopy (FLIM) to measure the fluorescence lifetimes of three different molecular rotors, in order to determine the microscopic viscosity in two model systems with significant biological interest. First, the constituents of a novel protocell - a model of a prebiotic cell - were studied using the molecular rotors BODIPY $\mathrm{C}_{10}$ and kiton red. Second, amyloid formation was investigated using the molecular rotor Cy3.
\end{abstract}

Key words: molecular rotor, fluorescence, fluorescence lifetime, FLIM, viscosity, confocal microscopy, protocells, protein aggregation, amyloid, lysozyme, neurodegeneration.

\section{INTRODUCTION}

Diffusion is an important process in nature as it can determine rates of reactions in many biological systems. Rates of diffusion are strongly affected by the viscosity of the surrounding environment and, as such, there is significant interest in developing techniques that permit measurement of viscosity on the micro-scale. Several fluorescence based techniques have been used including fluorescence anisotropy imaging [1] and fluorescence recovery after photobleaching (FRAP) [2]. In this article, we discuss an alternative fluorescent technique, namely optical imaging of fluorescent 'molecular rotors'.

Molecular rotors are fluorophores whose fluorescence quantum yield is dependent upon the viscosity of their immediate environment [3,4]. Thus, it is possible to calibrate the response of the fluorescence intensity or lifetime of a molecular rotor to viscosity by measuring the fluorescence in solutions of known viscosity. Subsequently, fluorescence measurements can then be used to extract the viscosity from samples where the viscoelastic properties are otherwise unknown, for example, in cells [5] or in encapsulated microbubbles [6]. In this article we use measurements of the fluorescence lifetime to study the viscosity trends and behaviour in two example biological systems. Firstly, a novel design for a protocell (a model for a prebiotic cell) is investigated. Secondly, aggregation of the protein lysozyme was studied due to the relevance of protein aggregation to neurodegenerative diseases.

As stated above, the fluorescence quantum yield of a molecular rotor depends upon the viscosity (or the degree of molecular crowding) in the vicinity of the rotor. Once excited, a molecular rotor can relax back to its ground electronic configuration in one of two ways: either by emission of a fluorescence photon or through intramolecular rotation. In viscous media the intramolecular rotation is hindered and fluorescence becomes the preferential decay pathway. This leads to a decrease in the non-radiative decay rate and a concomitant increase in the quantum yield $(\phi)$. The quantum yield is then dependent on the viscosity $(\eta)$ according to,

Imaging, Manipulation, and Analysis of Biomolecules, Cells, and Tissues XII, edited by

Daniel L. Farkas, Dan V. Nicolau, Robert C. Leif, Proc. of SPIE Vol. 8947, 89471C

(C) 2014 SPIE · CCC code: 1605-7422/14/\$18 - doi: 10.1117/12.2037851 
$\varphi=z \eta^{\alpha}$

where $z$ and $\alpha$ are constants of proportionality [7]. The fluorescence lifetime $\left(\tau_{f}\right)$ is related to the quantum yield according to,

$\varphi=\frac{k_{r}}{k_{r}+k_{n r}}=k_{r} \tau_{f}$

where $k_{r}$ and $k_{n r}$ are the radiative and non-radiative decay rates respectively. Thus, equation (1) can be written in terms of the fluorescence lifetime as,

$\tau_{f}=\frac{z \eta^{\alpha}}{k_{r}}$

Taking logarithms of both sides of equation (3) produces

$\log \left(\tau_{f}\right)=\log \left(\frac{Z}{k_{r}}\right)+\alpha \log (\eta)$

and it is now clear that the logarithms of fluorescence lifetime and viscosity are linearly related, allowing simple conversion between the two. Finally, it is worth noting that in the experiments presented here we have chosen to monitor the fluorescence lifetime of the molecular rotor as this circumvents problems associated with fluorescence intensity measurements, such as photobleaching, variations in excitation power and changes in fluorophore concentration.

In this article we present the application of molecular rotors to two biological experiments. Firstly, a novel design for a protocell was studied. A protocell is any model system for a prebiotic cell and, in this case, we used molecular rotors to investigate hybrid protocells consisting of a polyelectrolyte/ribonucleotide core (known as coacervates, these simulate a reaction centre) surrounded by a multilamellar oleic acid coating (which simulates a cell membrane). We used molecular rotors to monitor the viscosity of each constituent of the protocells - i.e. the coacervate core and the oleic acid membrane - individually. Further work involving the study of the fully formed protocells is presented in reference [8].

Secondly, the formation of amyloid through aggregation of the protein lysozyme was investigated because of its relevance to neurodegeneration and, in particular, to the disease systemic amyloidosis $[9,10]$. Amyloid formation through protein aggregation is typically studied using the fluorescent dye Thioflavin $T(T h T)$, which fluoresces when bound to aggregated (polymeric) protein but not in the presence of monomeric protein [11]. Thus, using ThT, it is possible to follow the progress of protein aggregation and to determine when aggregation is complete (when the rate of change of the ThT fluorescence intensity reaches zero). However, this method does not reveal any mechanical properties of the amyloids formed, nor does it provide information about the amount of amyloid produced. Here we used a molecular rotor to probe protein aggregation as this has the potential to provide quantitative measurements of the mechanical properties of amyloids (i.e. the viscosity) and, hence, to permit comparison of amyloid materials formed through the aggregation of different proteins.

\section{EXPERIMENTAL PROCEDURES}

\subsection{Protocells: measurements of sodium oleate micelles and PDDA/ATP coacervates}

In the protocell study, we used molecular rotors to investigate the viscosity of the two constituents of a hybrid protocell design. The first component is the coacervate core, which is a spontaneously formed polymer/ribonucleotide microdroplet. Coacervate dispersions were generated using an aqueous mixture of the polymer polydiallyldimethylammonium chloride (PDDA, $\mathrm{M}_{\mathrm{w}}=150 \mathrm{kda}$, monomer unit, $161.8 \mathrm{~g} \mathrm{~mol}^{-1}$ ) and adenosine triphosphate (ATP) at concentrations of $5 \mathrm{mM}$ PDDA and $1.25 \mathrm{mM}$ ATP. The viscosity of the coacervates was studied 
using the molecular rotor kiton red (sulforhodamine B, S1402, Sigma Aldrich) as this was readily soluble in the PDDA/ATP matrix.

The second consitutent of the protocells is the oleic acid membrane and to study this region we produced aqueous dispersions of sodium oleate micelles at a concentration of $20 \mathrm{mM}$. In this case, the molecular rotor BODIPY $\mathrm{C}_{10}$ (mesosubstituted 4,4-difluoro-4-bora-3a,4a-diaza-s-indacene) was used to sense viscosity as it was seen to be considerably more soluble in the oleate micelles than kiton red.

After preparing the coacervate or oleate micelle dispersions, the appropriate molecular rotor was added (see above) and the samples were then left to equilibrate for 10 minutes prior to imaging. The final concentrations of kiton red or BODIPY $\mathrm{C}_{10}$ were approximately $3 \mu \mathrm{M}$ in all samples and the structures of the two molecular rotors are shown in figure 1. All samples were imaged using the multiphoton microscope described in section 2.3 with excitation at $840 \mathrm{~nm}$ and emission recorded over the range 480-600 nm (BODIPY $\mathrm{C}_{10}$ ) or 500-700 nm (kiton red). It should be noted that the protocell experiments presented here are described in more detail in reference [8].

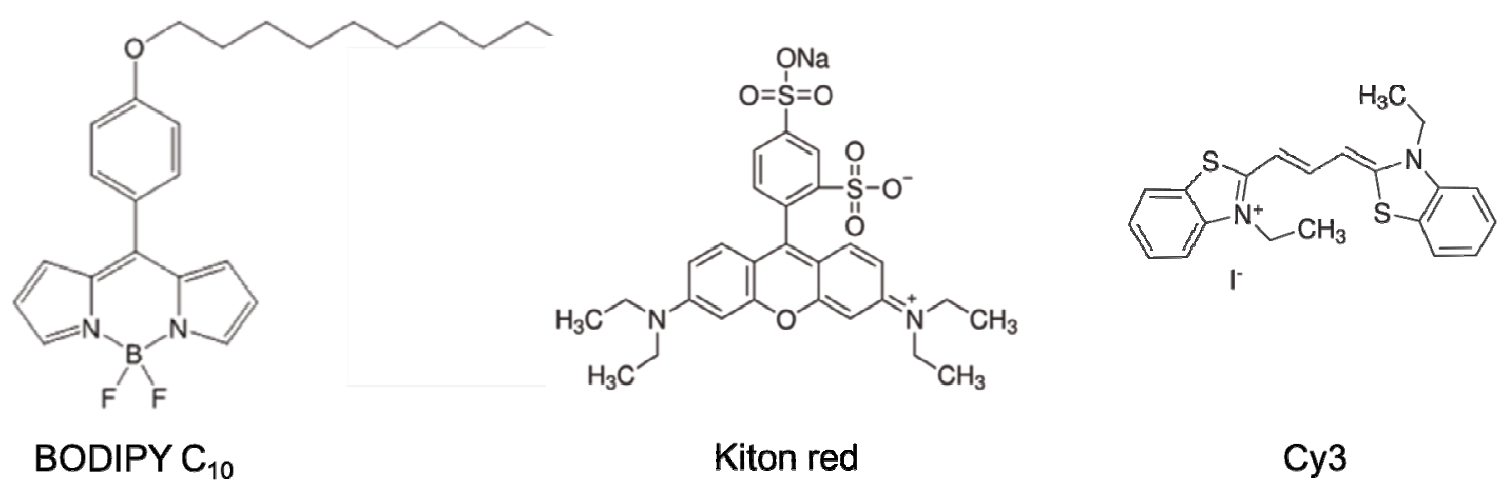

Figure 1. Molecular structures of the three fluorescent molecular rotors used in this study: BODIPY C10, kiton red and Cy3.

\subsection{Lysozyme aggregation}

Lysozyme aggregation time courses were performed using monomeric hen egg lysozyme in aqueous solution $(4 \mathrm{mg} / \mathrm{ml}$ lysozyme, $50 \mathrm{mM} \mathrm{HCl}, 100 \mathrm{mM} \mathrm{NaCl}, 0.2 \% \mathrm{v} / \mathrm{v}$ DMSO $)$ passed through a syringe driven filter $(0.22 \mu \mathrm{m}$ pore size $)$ and seeded with $1 \% \mathrm{v} / \mathrm{v}$ sonicated preformed fibrils $(10 \mathrm{mg} / \mathrm{ml}$ lysozyme, $50 \mathrm{mM} \mathrm{HCl}, 100 \mathrm{mM} \mathrm{NaCl})$. Aggregation was monitored using both confocal fluorescence lifetime imaging of the molecular rotor Cy3 (3,3'-diethylthiacarbocyanine iodide, 36809, Sigma Aldrich; structure shown in figure 1; sample concentration $3 \mu \mathrm{M}$ ) and fluorescence spectroscopy of ThT (sample concentration $20 \mu \mathrm{M}$ ). During aggregation samples were incubated at $56^{\circ} \mathrm{C}$. Fluorescence lifetime images of $\mathrm{Cy} 3$ were recorded every 0.5-5 minutes with an acquisition time of 1 minute using the multiphoton microscope described in section 2.3 (excitation $960 \mathrm{~nm}$, emission 525-700 nm). ThT fluorescence intensity measurements were made at 1 minute intervals using a fluorometer, with excitation at $440 \mathrm{~nm}$ and emission monitored at $480 \mathrm{~nm}$.

\subsection{Fluorescence lifetime imaging and viscosity determination}

All imaging and fluorescence lifetime measurements presented herein were performed using a multiphoton microscope. This consisted of a confocal microscope (TCS SP5 II Confocal, Leica Microsystems GmbH, Germany) with an external Titanium:Sapphire laser (Chameleon Vision II, Coherent Ltd., UK), which provided pulsed excitation over the range 680-1080 nm permitting time-resolved fluorescence imaging with multiphoton excitation. All excitation and detection parameters were as described in sections 2.1 and 2.2. Fluorescence decays in all image pixels were fit to a monoexponential model using SPCImage fitting software (Becker \& Hickl GmbH, Germany) according to equation (5), 
$I(t)=I_{0} \exp \left(\frac{-t}{\tau_{f}}\right)$

where $I$ represents the fluorescence intensity, $t$ is the time and $I_{0}$ is the intensity at time $t=0$. The temporal instrument response function (IRF) was accounted for by recording the second harmonic generation signal from a sample of urea crystals, as this provides a close approximation of the IRF. Measured fluorescence lifetimes were converted to viscosities according to equation (3).

As discussed above, it is necessary to calibrate the fluorescence lifetimes of each of the molecular rotor dyes used. This was achieved by measuring fluorescence decays from $\mathrm{Cy} 3$, kiton red and BODIPY $\mathrm{C}_{10}$ in solutions of known viscosity and then plotting the fluorescence lifetime as a function of viscosity. For BODIPY $\mathrm{C}_{10}$ and $\mathrm{Cy} 3$ we used methanol:glycerol solutions of varying concentrations for the calibration experiments as the dyes dissolved easily in these solvents. For kiton red we used glycerol:water solutions of varying concentration as kiton red dissolved readily in water but not in methanol. The calibration data used for BODIPY $\mathrm{C}_{10}$ is presented in figure 2 [3, 6, 12]. Similar data were observed for $\mathrm{Cy} 3$ (data not shown) and kiton red [13].

(a)

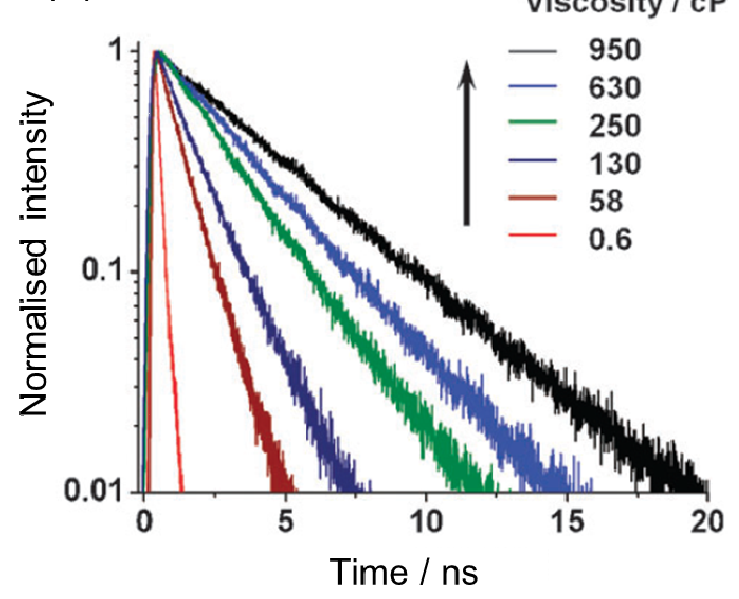

(b)

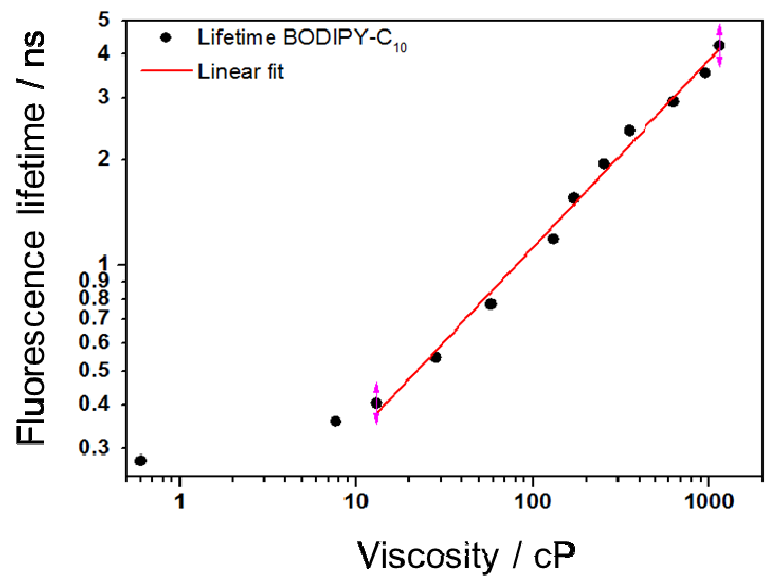

Figure 2. Viscosity calibration of BODIPY $\mathrm{C}_{10}$. (a) Fluorescence decays recorded in solvents of varying viscosity (methanol:glycerol mixtures) showing that the fluorescence lifetime increases with increasing solvent viscosity. (b) Log-log graph showing the variation in fluorescence lifetime with viscosity. We observe a linear relationship in the region 10-1000 cP, which facilitates simple conversion between measured fluorescence lifetimes and viscosities using equation (3). Data collated from references $[3,6,12]$.

\section{RESULTS AND DISCUSSION}

\subsection{Protocells}

Figure 3 shows fluorescence lifetime images of coacervates (a) and oleic acid micelles (b) stained with kiton red and BODIPY $\mathrm{C}_{10}$ respectively. The images show that we have successfully formed dispersions of coacervates and sodium oleate micelles, which is the first step in making the hybrid protocells consisting of a coacervate core surrounded by an oleic acid membrane. The mean kiton red fluorescence lifetime observed in the coacervates was $2270 \mathrm{ps,} \mathrm{which}$ translates to a viscosity of $2.77 \mathrm{cP}$. Similarly, the mean lifetime of BODIPY $\mathrm{C}_{10}$ observed in the Sodium oleate vesicles was $656 \mathrm{ps}$ corresponding to a viscosity of $38.6 \mathrm{cP}$. These data show that we are able to use molecular rotors to quantitatively measure viscosity in the two constituents of a model protocell. Our future aim is to monitor the viscosity in both the coacervate and oleate regions within the fully formed protocells using the same methods employed here. By measuring similar viscosities to those reported here, we hope to determine successful formation of the hybrid protocells. 

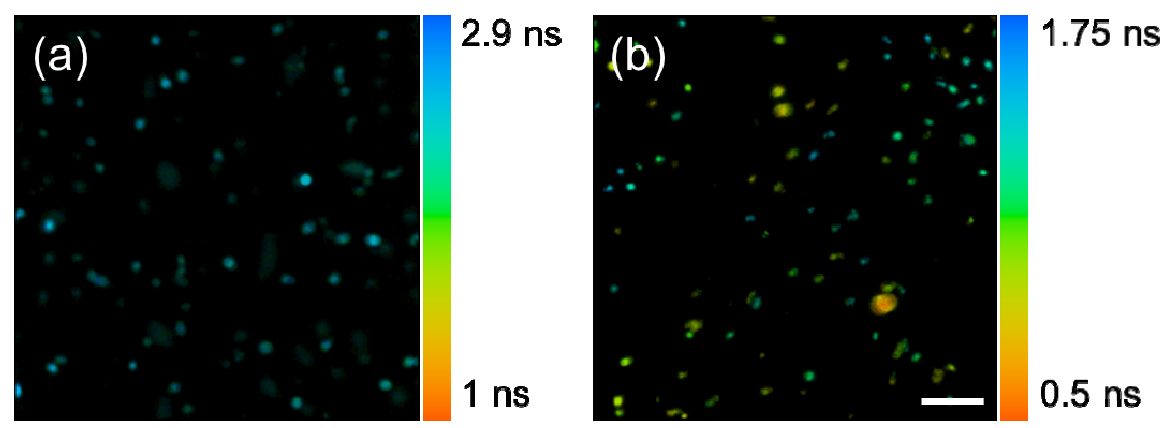

Figure 3. Fluorescence lifetime images of a coacervate dispersion stained with the molecular rotor kiton red (a) and a sodium oleate micelle dispersion stained with BODIPY $\mathrm{C}_{10}$ (b). Fluorescence lifetime is represented by the false colour scale adjacent to each image. Scale bar in (b) is $25 \mu \mathrm{m}$ in length and is applicable to both images.

\subsection{Lysozyme aggregation}

Using the molecular rotor $\mathrm{Cy} 3$ we monitored the aggregation of lysozyme. Figure 4 shows the fluorescence intensity and lifetime observed at several time points during the aggregation. It is clear from the images that amyloid fibrils formed during the aggregation and these were seen to become both more prevalent and more stable over time. The fluorescence lifetime increased during aggregation indicating that amyloid fibrils have a much higher viscosity than monomeric lysozyme. The aggregation was deemed to be complete after approximately 70 minutes, at which point the increase in the fluorescence lifetime had ceased and no more fibrils appeared to be forming. ThT fluorescence intensity assays were also conducted under the same conditions and these confirmed the rate of aggregation observed with Cy3 (data not shown). Thus, using the molecular rotor $\mathrm{Cy} 3$, it is possible to monitor the aggregation of lysozyme in a similar fashion to that used for ThT measurements. Importantly, the use of $\mathrm{Cy} 3$ provides the capability to quantitatively measure viscosity, which is not possible using ThT.
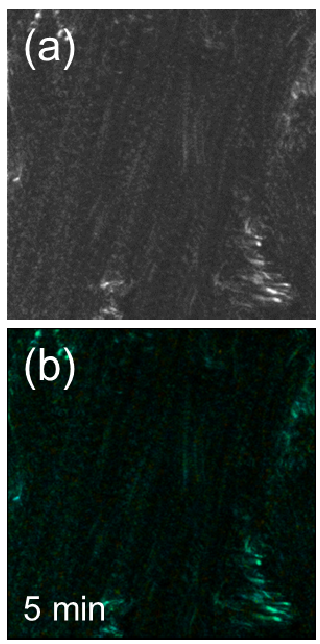
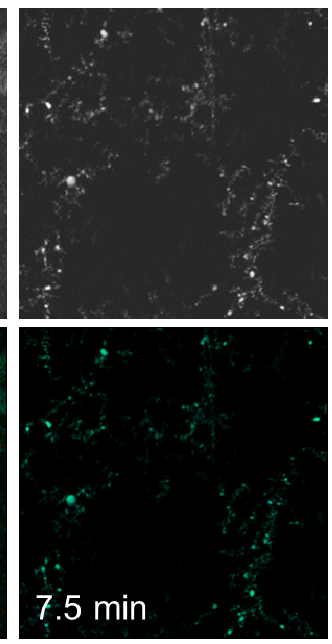
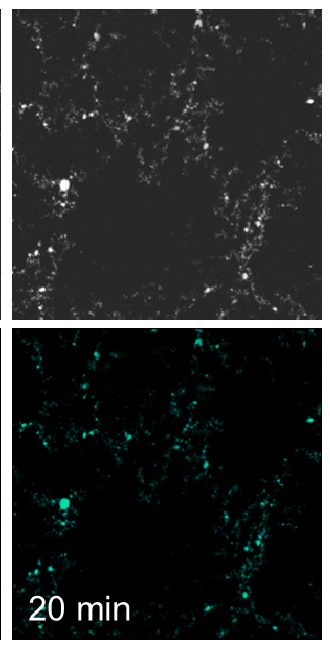
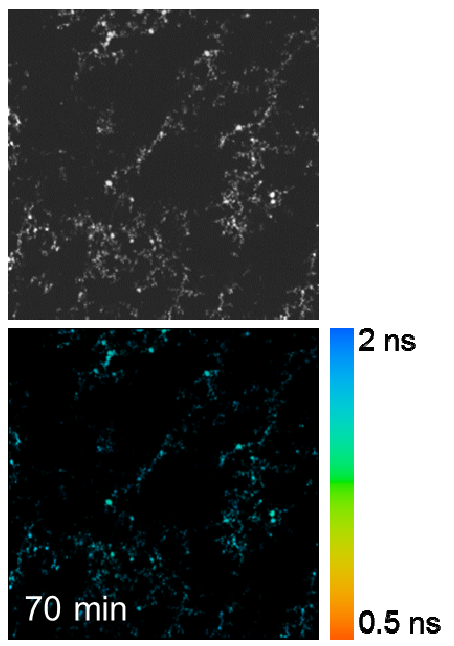

Figure 4. Fluorescence intensity (a) and lifetime (b) images of aggregating lysozyme. Times shown in the images indicate the time from the beginning of the aggregation (i.e. the time at which the aggregation was seeded with pre-formed fibrils). In (b) the fluorescence lifetime is represented by the false colour scale shown to the right of the images.

\section{CONCLUSIONS}

This article has discussed the use of fluorescent molecular rotors to quantitatively monitor viscosity in two separate biological experiments. In a study of a novel design for a protocell, the molecular rotors kiton red and BODIPY $\mathrm{C}_{10}$ were 
used to investigate the viscosity of the two constituents of the protocell. In this way it was possible to ascertain the viscosities in aqueous dispersions of both coacervates (which will form the core of the protocells) and sodium oleate micelles (which will form the membrane of the protocells). Further work will now aim to use identical measurements in the fully formed protocells to provide evidence for successful formation.

In a preliminary study of lysozyme aggregation we have shown that it is possible to follow the aggregation process using the molecular rotor $\mathrm{Cy} 3$. The timing of aggregation was in agreement with that observed using ThT, which is the standard dye used to monitor protein aggregation. Cy3 (and other molecular rotors) can provide quantitative measurements of viscosity and, hence, offer advantages over ThT in that they will permit determination of the mechanical properties of amyloid materials and will also allow comparison of the amyloid materials formed through the aggregation of different proteins. Further work will now involve more detailed investigation of the Cy3 fluorescence decays to ascertain whether they can also provide additional information about the aggregation process that is not available with ThT.

\section{ACKNOWLEDGMENTS}

The authors gratefully acknowledge the funding for this work, which was provided by the Engineering and Physical Sciences Research Council. Marina K. Kuimova acknowledges her EPSRC Career Acceleration Fellowship.

\section{REFERENCES}

[1] Suhling, K., Siegel, J., Lanigan, P. M., Leveque-Fort, S., Webb, S. E., Phillips, D., Davis, D. M., and French, P. M., "Time-resolved fluorescence anisotropy imaging applied to live cells," Opt Lett, 29(6): p. 584-6 (2004)

[2] Dayel, M. J., Hom, E. F., and Verkman, A. S., "Diffusion of green fluorescent protein in the aqueous-phase lumen of endoplasmic reticulum," Biophys J, 76(5): p. 2843-51 (1999)

[3] Kuimova, M. K., "Mapping viscosity in cells using molecular rotors," Phys Chem Chem Phys, 14(37): p. 12671$86(2012)$

[4] Haidekker, M. A. and Theodorakis, E. A., "Environment-sensitive behavior of fluorescent molecular rotors," Journal of biological engineering, 4( ): p. 11 (2010)

[5] Kuimova, M. K., Botchway, S. W., Parker, A. W., Balaz, M., Collins, H. A., Anderson, H. L., Suhling, K., and Ogilby, P. R., "Imaging intracellular viscosity of a single cell during photoinduced cell death," Nature Chemistry, 1(1): p. 69-73 (2009)

[6] Hosny, N. A., Mohamedi, G., Rademeyer, P., Owen, J., Wu, Y., Tang, M. X., Eckersley, R. J., Stride, E., and Kuimova, M. K., "Mapping microbubble viscosity using fluorescence lifetime imaging of molecular rotors," Proc Natl Acad Sci U S A, 110(23): p. 9225-30 (2013)

[7] Forster, T. and Hoffmann, G., "Viscosity dependence of fluorescent quantum yields of some dye systems," Zeitschrift Fur Physikalische Chemie-Frankfurt, 75(1-2): p. 63 (1971)

[8] Tang, T.-Y. D., Che Hak, C. R., Thompson, A. J., Kuimova, M. K., Williams, D. S., Perriman, A. W., and Mann, S., "Fatty acid membrane assembly on coacervate micro-droplets as a step towards a hybrid protocell model," Nature Chemistry, (in review)

[9] Pepys, M. B., Hawkins, P. N., Booth, D. R., Vigushin, D. M., Tennent, G. A., Soutar, A. K., Totty, N., Nguyen, O., Blake, C. C., Terry, C. J., and et al., "Human lysozyme gene mutations cause hereditary systemic amyloidosis," Nature, 362(6420): p. 553-7 (1993)

[10] Booth, D. R., Sunde, M., Bellotti, V., Robinson, C. V., Hutchinson, W. L., Fraser, P. E., Hawkins, P. N., Dobson, C. M., Radford, S. E., Blake, C. C. F., and Pepys, M. B., "Instability, unfolding and aggregation of human lysozyme variants underlying amyloid fibrillogenesis," Nature, 385(6619): p. 787-793 (1997)

[11] LeVine, H., 3rd, "Thioflavine t interaction with synthetic alzheimer's disease beta-amyloid peptides: Detection of amyloid aggregation in solution," Protein Sci, 2(3): p. 404-10 (1993)

[12] Levitt, J. A., Kuimova, M. K., Yahioglu, G., Chung, P. H., Suhling, K., and Phillips, D., "Membrane-bound molecular rotors measure viscosity in live cells via fluorescence lifetime imaging," Journal of Physical Chemistry C, 113(27): p. 11634-11642 (2009)

[13] Hosny, N. A., Fitzgerald, C., Tong, C., Kalberer, M., Kuimova, M. K., and Pope, F. D., "Fluorescence lifetime imaging of atmospheric aerosols: A direct probe of aerosol viscosity," Faraday Discussions, 165(1): p. 343-356 (2013) 